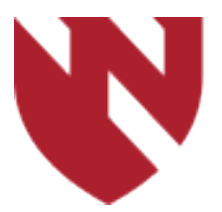

December 2019

\title{
Environmental Exposures and Respiratory Health
}

\author{
Letisha J. Ferris \\ University of Nebraska Medical Center \\ Corrine K. Hanson \\ University of Nebraska Medical Center \\ Joan Lappe \\ University of Nebraska Medical Center \\ Nancy Waltman \\ University of Nebraska Medical Center \\ Laura Bilek \\ University of Nebraska Medical Center
}

See next page for additional authors

Tell us how you used this information in this short survey.

Follow this and additional works at: https://digitalcommons.unmc.edu/gmerj

Part of the Higher Education Commons, and the Medicine and Health Sciences Commons

\section{Recommended Citation}

Ferris, L. J., Hanson, C. K., Lappe, J., Waltman, N., Bilek, L., , Poole, J. A. Environmental Exposures and Respiratory Health. Graduate Medical Education Research Journal. 2019 Dec 13; 1(1).

https://digitalcommons.unmc.edu/gmerj/vol1/iss1/59

This Conference Proceeding is brought to you for free and open access by DigitalCommons@UNMC. It has been accepted for inclusion in Graduate Medical Education Research Journal by an authorized editor of DigitalCommons@UNMC.For more information, please contact digitalcommons@unmc.edu. 


\section{Environmental Exposures and Respiratory Health}

Creative Commons License

(c) (i) (9)

This work is licensed under a Creative Commons Attribution-Noncommercial-No Derivative Works 4.0 License.

Authors

Letisha J. Ferris, Corrine K. Hanson, Joan Lappe, Nancy Waltman, Laura Bilek, and Jill A. Poole 
infusion and fibrate prior to discharge. Elevated triglycerides should always be considered when determining the etiology of acute pancreatitis, even in patients who consume alcohol as excessive alcohol consumption can lead to a state of hypertriglyceridemia. This case also illustrates the interesting relationship between elevated serum lipids and measured serum electrolytes. The presence of elevated lipids increases the non-aqueous components of the serum with a concomitant decrease in the fraction of serum composed of free water and electrolytes. This phenomenon, termed the "electrolyte exclusion effect", is common with the use of flame photometry or indirect potentiometry, the methods utilized by many hospital laboratories. However, this artifact does not occur with the use of direct potentiometry, as this method directly measures the concentration of electrolytes in the water phase of serum. Point-of-caretesting is more accurate in the setting of severe hypertriglyceridemia as it utilizes direct ion-selective electrodes. This can be used in the setting of hypertriglyceridemia or hyperproteinemia to obtain more precise results.

https://doi.org/10.32873/unmc.dc.gmerj.1.1.056

\section{A Cough May be Just the Tip of the Iceberg}

Ian Cormier, Neil Bhogal, Michael Smith

\section{Mentor: Michael Smith \\ Program: Internal Medicine}

Pyogenic liver abscesses (PLAs) can be diagnostically challenging, as they may present with non-specific symptoms and are less frequently encountered than other types of infections. PLAs caused by atypical organisms may be rare and can be associated with a variety of other underlying pathologies including occult malignancy.

A middle-aged patient presented with two weeks of productive cough, dyspnea, subjective fevers and chills. The patient was started on antibiotics for community acquired pneumonia but decompensated with signs of sepsis. Computed tomography (CT) of the chest, abdomen and pelvis revealed multiple bilateral pulmonary nodules with early cavitation concerning for septic emboli and a large multiloculated hepatic hypodensity in the right lobe of the liver concerning for a liver abscess. Anaerobic cultures from an aspirate of the lesion grew Fusobacterium nucleatum. The patient was discharged on intravenous antibiotics along with an outpatient colonoscopy to evaluate for occult malignancy. PLAs, which are typically polymicrobial, are the most common type of liver abscess in the U.S. Many PLAs result from portal vein pyemia, usually related to bowel leakage and peritonitis. Biliary infection and hematogenous spread are other common etiologies. Fusobacterium spp. are anaerobic gram negative rods that occur as normal oral flora and have been associated with periodontal infections. However, they are uncommon causes of pyogenic liver abscesses. PLAs have a known association with GI malignancies and Fusobacterium nucleatum has recently been shown to promote colorectal carcinogenesis by affecting genes associated with the adenoma-carcinoma sequence. The oral cavity could be a potential source of infection for this patient. However, occult GI malignancy must be ruled out, as there is an association between PLAs and malignancy of the gastrointestinal tract. https://doi.org/10.32873/unmc.dc.gmerj.1.1.057

\section{Efficacy of Endoscopic Ultrasonography Guided Gastroenterostomy in Gastric Outlet Obstruction Dhaliwal Amaninder, Cormier lan, Jhand Aravdeep, Rangray Rajani, Bhat Ishfaq, Girotra Mohit, Singh Shailender}

Mentor: Amaninder Dhaliwal

Program: Internal Medicine

Introduction: Gastric outlet obstruction (GOO) is the complication of benign and malignant diseases. Laproscopic gastrojejunostomy and enteral stenting has been the standard of care. Endoscopic ultrasonography guided gastroenterostomy (EUS-GE) has emerged as a newer technique in patients with GOO. Thus, we conducted systematic review and meta-analysis to report the overall technical success, clinical success and adverse events.

Methods: Multiple electronic databases (MEDLINE, EMBASE and Google
Scholar) and conference abstracts were comprehensively searched to identify studies reporting EUS-GE in patients with GOO. References from identified studies were then manually searched for any additional studies. Primary outcome of our meta-analysis was to see the overall pooled technical and clinical success of the procedure. Secondary outcome was to look at the total adverse events of the procedure. The meta-analysis was performed using Der Simonian and Laird random effect model.

Results: 10 studies were included in the final meta-analysis reporting a total of 263 patients (males 135 and females 128) with median age of 61.2 years. The median follow up was 110 days. Overall pooled technical and clinical success rates of the procedure were $92.4 \%$ $(95 \% \mathrm{CI}=89-95, \mathrm{I} 2=0)$ and $88.5 \%(95 \% \mathrm{CI}=$ $84.5-92.5, \mathrm{I} 2=0)$. The total adverse events were $8.1 \%(95 \% \mathrm{CI}=4.1-11.9, \mathrm{I} 2=23 \%)$ mainly as stent migration $(\mathrm{n}=2)$ and peritoneal stent deployment $(\mathrm{n}=15)$.

Conclusion: EUS-GE is a safe and effective technique in patients with GOO with overall high technical and clinical success rates (92.4\%, 88.5\% respectively). However, further randomized control trials are needed to validate these findings.

https://doi.org/10.32873/unmc.dc.gmerj.1.1.058

\section{Environmental Exposures and Respiratory Health}

\section{Letisha J Ferris, Corrine K Hanson; Joan Lappe; Nancy Waltman; Laura Bilek; Jill A Poole}

Mentors: Jill A. Poole and Corrine K. Hanson

Program: Internal Medicine

Background and Objectives: Chronic respiratory diseases lead to co-morbid conditions, including osteoporosis, and environmental exposures are risk factors for respiratory disease development and severity. We evaluated the relationship between environmental exposures and respiratory health in postmenopausal women to better understand how this relationship might impact postmenopausal health outcomes.

\begin{abstract}
Methods: A cross-sectional, sub-study of participants of the Heartland Osteoporosis Prevention Study was conducted whereby questionnaires that included information on agriculture exposures and respiratory health symptoms and diagnoses were mailed and
\end{abstract}


collected. Descriptive data and trends were analyzed using SPSS software.

Results: 233 female participants provided the requested information. Over $20 \%$ of the participants endorsed significant agriculture exposure defined as greater than or equal to 2 years exposure that occurred predominately during youth and young adulthood. Exposure included both livestock and crops, with the most common exposures being corn, soybeans, and beef cattle. In addition, $55 \%$ reported significant second-hand smoke exposure during youth. More than half (53\%) reported at least one respiratory disease, which included allergic rhinoconjuntivitis $(45 \%)$, sinusitis $(10 \%)$, asthma $(6 \%)$, chronic obstructive airway disease $(2 \%)$, and pneumonia (14\%).
Conclusions: Agriculture and second-hand smoke exposures were common early life exposures in post-menopausal women of the Heartland Osteoporosis cohort that could impact development of respiratory diseases. Future analysis will investigate how environmental exposures and respiratory diseases influence post-menopausal health and bone health.

https://doi.org/10.32873/unmc.dc.gmerj.1.1.059

\section{Assessment of Treatment Response using the Multi-Biomarker Disease Activity Score in Rheumatoid Arthritis Patients Initiating Methotrexate}

Tate Johnson, Bryant England, Harlan Sayles, Geoffrey Thiele, Ted Mikuls, James O’Dell

Mentor: Bryant England

Program: Internal Medicine

Background/Purpose: The Multi-Biomarker Disease Activity (MBDA) score was developed to provide an objective measure of rheumatoid arthritis (RA) disease activity. The ability of the MBDA to predict treatment response may vary among disease-modifying agents, and has not been evaluated in patients initiating methotrexate (MTX), a cornerstone therapy in RA.

Methods: We conducted a secondary analysis of an open-label study of RA patients initiating MTX. The association of MBDA scores at baseline $(\mathrm{N}=130)$ and week 16 $(\mathrm{N}=95)$ with treatment response was assessed using multivariable regression models. Convergent validity and responsiveness were determined by calculating correlations of the MBDA with disease activity measures and through the calculation of standardized response means (SRM).

Results: Patients achieving an ACR50 response demonstrated greater reductions in MBDA than those failing to achieve an ACR50 response $(p=0.01)$. However, baseline MBDA scores (OR 1.01, 95\% CI 0.99, 1.04) and categories (moderate: OR 1.55, 95\% CI 0.45-14.20; high: OR 2.55, 95\% CI 0.5012.98) were not predictive of response. Higher baseline MBDA scores were associated with greater improvement in DAS28-ESR $(\mathrm{p}=0.01)$, as were baseline DAS28-ESR values $(\mathrm{p}<0.001)$. The MBDA demonstrated moderate correlations with DAS28-ESR and ESR, but weaker correlations with the HAQ and PtGA. Treatment responsiveness was greater for DAS28-ESR (SRM -1.32) than MBDA (SRM -0.65).

Conclusions: Baseline MBDA was associated with greater reductions in DAS28-ESR but was not a robust predictor of ACR50 response. Although demonstrating moderate convergent validity with standard disease activity assessments, the MBDA yielded lower responsiveness than the DAS28-ESR in the context of MTX treatment.

https://doi.org/10.32873/unmc.dc.gmerj.1.1.060

\section{Nonspecific Interstitial Pneumonia Development after Fire Extinguisher Dust Inhalation \\ Maureen McElligott and Daniel Hershberger}

Mentor: Daniel Hershberger

Program: Internal Medicine

Introduction: Nonspecific interstitial pneumonia (NSIP) is a form of chronic inflammatory idiopathic interstitial pneumonia. It typically presents with months of dyspnea. NSIP is frequently associated with connective tissue disease, HIV, and druginduced lung disease.

Case Presentation: A middle-aged patient, non-smoker with hypertension and OSA on CPAP presented with six months of exertional dyspnea and right-sided chest pain. The patient developed a new exertional 10 LPM supplemental oxygen requirement after significant smoke and fire extinguisher dust inhalation. The patient denied a family history of lung disease or connective tissue disease, had faint inspiratory crackles, and no leg edema.

ANA, RF, ANCA, IgE, CK, aldolase, anti$\mathrm{CCP}, \mathrm{HIV}$, and hypersensitivity pneumonitis panel were unremarkable. Chest CT revealed diffuse ground glass sparing the costophrenic angles without traction bronchiectasis and honeycombing. Pulmonary function testing revealed FEV1/FVC of $82 \%$, TLC was $56 \%$ predicted, and DLCO was $63 \%$ predicted. EF was normal and pulmonary artery systolic pressure was $45-50 \mathrm{mmHg}$. Right lower lobe wedge biopsy demonstrated alveolar wall thickening with focal lymphocytic inflammation in a fairly uniform distribution without honeycombing, consistent with NSIP. The patient underwent pulmonary rehabilitation and was started on Prednisone and transitioned to Azathioprine resulting in improvement of his dyspnea.

Discussion: Diagnosis of NSIP is dependent on multidisciplinary interactions among pulmonology, radiology, and pathology to differentiate it from other interstitial lung diseases and guide treatment. The patient developed hypoxic dyspnea within months of fire extinguisher dust exposure and lacked other risk factors for development of NSIP. Fire extinguisher exposure is typically associated with desquamative interstitial pneumonia, and per our literature review, there are no reported cases of fire extinguisher exposure related to NSIP, making this case unique.

Conclusion: It is probable that exposure to smoke and fire extinguisher dust inhalation triggered the development of NSIP.

https://doi.org/10.32873/unmc.dc.gmerj.1.1.061 